\title{
Mono- and Biexponential Luminescence Decays of Individual Single-Walled Carbon Nanotubes
}

\author{
Tobias Gokus,${ }^{\dagger}$ Laurent Cognet, ${ }^{\dagger}$ Juan G. Duque, ${ }^{\dagger, \S, \|}$ Matteo Pasquali, ${ }^{\S}$ Achim Hartschuh, ${ }^{\dagger}$ and \\ Brahim Lounis*,
}

Centre de Physique Moléculaire Optique et Hertzienne, Université de Bordeaux and CNRS, 351 Cours de la Libération, Talence F-33405, France, Department Chemie und Biochemie and CeNS,

Ludwig-Maximilians-Universität München, 81377 München, Germany, and Department of Chemical and Biomolecular Engineering, Rice University, 6100 Main Street, Houston, Texas 77005

Received: May 28, 2010; Revised Manuscript Received: July 9, 2010

\begin{abstract}
We have studied the exciton recombination dynamics of individual $(6,4)$ and $(6,5)$ single-walled carbon nanotubes embedded in aqueous gels or deposited on glass surfaces. CoMoCat nanotubes systematically display short monoexponential photoluminescence (PL) decays presumably due to defects introduced during their synthesis. In contrast HiPco nanotubes can either display mono- or biexponential PL decays depending on the environmental conditions. Transition from bi- to monoexponential decays can be reproduced by a simple three level model taking into account defect-dependent nonradiative decay mechanisms.
\end{abstract}

The photoluminescence (PL) properties of semiconducting single walled carbon nanotubes (SWNTs) have attracted much attention over the last years. ${ }^{1}$ These properties strongly depend on the structure of each nanotube $e^{2}$ but also on extrinsic factors resulting for instance from synthesis or environmental factors. ${ }^{3-7}$ As a result, PL studies performed on ensemble of SWNTs are affected by inhomogeneities that hinder the development of a detailed understanding of the underlying mechanisms. Experiments on individual SWNTs remove part of this heterogeneity by allowing description of the characteristics of each SWNT in its particular environment. ${ }^{8}$ Comparisons between values of a physical parameter extracted from different experimental reports are however difficult because, generally, the studied samples differ by synthesis methods or preparation procedures. For example PL decays performed on individual $(6,4)$ and $(6,5)$ nanotubes by different groups have resulted in distinct behaviors, ranging from very short monoexponential decays to longer biexponential ones. $5,7,9-11$

This paper aims at understanding this large disparity in PL decay behaviors. We experimentally confirm that PL decays of $(6,5)$ and $(6,4)$ nanotubes can exhibit either mono- or biexponential behaviors and show that these depend on synthesis methods and nanotube environment. These observations are explained by a previous simple three-level model ${ }^{10}$ taking now into account the defect-dependent dominant nonradiative decay mechanisms proposed by Pereibenos et al. ${ }^{12}$

The SWNTs used in this study were either synthesized using HiPco or CoMoCat methods. The nanotubes were dispersed in aqueous suspensions of the anionic surfactants sodium deoxycholate (DOC). For observations, single-molecule wide-field and confocal PL microscopes were used to image SWNTs excited with a continuous wave laser. The SWNTs were immobilized

* To whom correspondence should be addressed. E-mail: blounis@ u-bordeaux1.fr.

† Centre de Physique Molé de Bordeaux and CNRS.

* Ludwig-Maximilians-Universität München.

§ Rice University.

"Present address: Los Alamos National Laboratory, Los Alamos, NM 87544. in aqueous agarose gels (5 wt \%) or spin-coated on surfaces. SWNTs concentration was kept well below $1 \mu \mathrm{m}^{-3}$ such that bright individual nanotubes could be optically resolved. The PL of these bright tubes was sent to a spectrometer for further spectral identifications of individual $(6,4)$ or $(6,5)$ nanotubes. Typically, isolated SWNTs feature narrow PL lines from their bright excitonic state $E_{11}$, with full width at half-maximum (fwhm) in the range of $\sim 17-22 \mathrm{~nm}$. Following identification of these bright individual nanotubes, the excitation modality was switched to pulsed excitation $(\sim 150$-fs pulses, $76-\mathrm{MHz}$ repetition rate) using either a Ti:Sa oscillator (for excitation of a vibrational band of the first optical transition at $\sim 800 \mathrm{~nm}$ or $\sim 810 \mathrm{~nm}$ ) or an optical parametric oscillator (for resonant excitation at the second optical transition at $\sim 570 \mathrm{~nm}$ ). The PL decays were then recorded using conventional time-correlated single-photon counting (TCSPC) setups. Narrow bandpass filters (fwhm $=20 / 10 \mathrm{~nm}$ ) centered at the peak wavelength of the PL in front of the avalanche photo diode were used to suppress spurious spectral contributions like, e.g., scattered laser light. The decays were obtained in $10 \mathrm{~min}$ integration time with low excitation intensities $\leq 10^{12}$ photons $/\left(\right.$ pulse $\left.\cdot \mathrm{cm}^{2}\right)$ to ensure that less than one photon is absorbed per pulse knowing the resonant absorption cross-section of the SWNTs $\left(\sim 10^{-17} \mathrm{~cm}^{2}\right.$ per carbon atom). ${ }^{10,13}$

For data fitting we record the instrumental response function (IRF) of the TCSPC setup at a wavelength close to the peak emission line ( $~ 980 \mathrm{~nm}$ for $(6,5)$ and $\sim 880 \mathrm{~nm}$ for $(6,4))$ of the nanotubes (see Supporting Information). This is an important point since the penetration depth of light into the photoactive Si layer of avalanche photodiodes is wavelength dependent and measuring the IRF at the excitation wavelength (close to $E_{22}$ ) would lead to an erroneous additional decay component as reported $^{14}$ for CoMoCat tubes.

Figure 1a shows two representative luminescence decays of $(6,5)$ SWNTs synthesized by HiPco and CoMoCat methods studied in agarose gels. The two recorded PL transients are strikingly different. A long time component is observed in the HiPco nanotube, while it is completely absent in the CoMoCat one. A least-squares fitting algorithm was used to extract the 


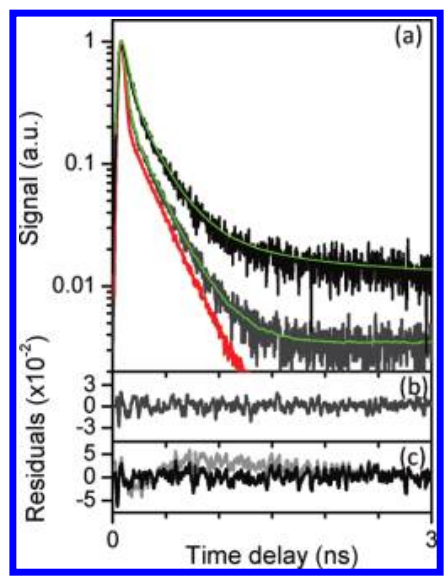

Figure 1. (a) PL decays of $(6,5)$ SWNTs dispersed in DOC and studied in aqueous gels. The nanotubes were excited at their second order excitonic resonance $(567 \mathrm{~nm})$. Black, HiPco nanotube; gray, CoMoCat nanotube; red, instrumental response function. The green curves are fits using biexponential (HiPco) or monoexponential (CoMoCat) curves. The weighted residuals defined as $[\operatorname{signal}(t)-\operatorname{fit}(t)] / \operatorname{signal}(t)^{1 / 2}$ are shown in (b) for CoMoCat. For HiPco (c), the weighted residuals for a monoexponential fit are also displayed in light gray for comparison and the corresponding $\chi^{2}$ is degraded by more than $40 \%$ in comparison to the biexponential fit $\left(7 \times 10^{-4}\right.$ vs $\left.10^{-3}\right)$.

decay times and decay dynamics from the transients. The weighted residuals and the corresponding "reduced chi-squared" parameter $\chi^{2}$ (see caption of Figure 1) are a measure for the quality of the fits and are used to distinguish mono- and biexponential decay behaviors. Data fitting of the PL decays reveals that CoMoCat SWNTs studied in aqueous gels systematically display monoexponential behaviors as previously reported on glass surfaces ${ }^{5}$ (Figure 1b). In contrast, bright HiPco SWNTs recorded in aqueous gels systematically display biexponential decays as previously reported ${ }^{10}$ (Figure 1c). Parts a and $\mathrm{b}$ of Figure 2 shows that the long time component $\tau_{\text {long }}$ of the PL decays ranges from 0.4 to 2 ns while the short time component $\tau$ ranges from 35 to $60 \mathrm{ps.} \mathrm{The} \mathrm{latter} \mathrm{is} \mathrm{significantly}$ larger than the decay time of CoMoCat nanotubes $(\tau=10-30$ ps, Figure 2d).

To investigate the influence of the nanotube environments, PL decays from HiPco nanotubes suspended in DOC and spincoated onto bare coverslips were also measured. In this case, the PL decays bridge the two behaviors obtained for CoMocCat and HiPco nanotubes in aqueous gels (parts a and c of Figure 2). Indeed, half of the SWNTs studied on glass surfaces displays a monoexponential behavior with decay times ranging from 10 to $40 \mathrm{ps}$ while the other half displays biexponential decays with $\tau$ ranging from $\sim 30-50 \mathrm{ps}$ and $\tau_{\text {long }}$ centered at $\sim 450 \mathrm{ps}$. This broad range of behavior clearly reflects the heterogeneous environment of the nanotubes lying on glass coverslips. ${ }^{15}$ Interestingly, the long time component fractional yield $A_{\text {long }}$ decreases with $\tau_{\text {long }}$ (Figure 2e), and when the fast components

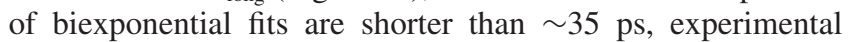
decays are in general satisfactorily fitted by monoexponential curves.

We now consider $(6,4)$ HiPCo nanotubes embedded in agarose gel or deposited on glass. In contrast to $(6,5)$ nanotubes, most of the recorded decays are monoexponential. Indeed, only few nanotubes in gels display biexponential decays ( 4 over 42 studied tubes) and none on surface. Figure 3 shows that monoexponential decays in agarose gel vary between 30 and $80 \mathrm{ps}$ with an average value of $52 \mathrm{ps}$. On glass the distribution is shifted toward smaller values with an average decay time of

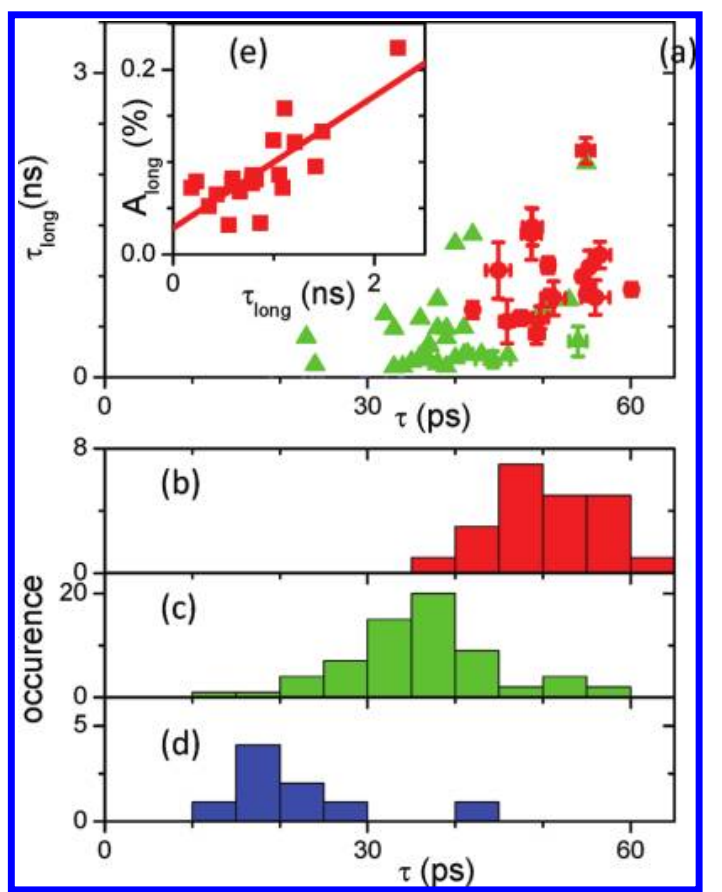

Figure 2. PL decays of $(6,5)$ SWNTs: (a) Long decay times $\tau_{\text {long }}$ as a function of short ones, $\tau$, deduced from the biexponential PL decays of individual HiPco SWNTs studied in aqueous gels (red) or on surfaces (green). (b) Histogram of the short time $\tau$ for HiPco $(6,5)$ SWNTs studied in aqueous gels (all of them displayed biexponential PL decays). (c) Histogram including both the short time $\tau$ for HiPco SWNTs studied on surfaces displaying biexponential PL decays and the single decay time $\tau$ for those displaying monoexponential decays (50\% of total). (d) Histogram of the decay time $\tau$ for CoMoCat (all of them displayed monoexponential decays). (e) Long time component fractional yield $A_{\text {long }}$ as a function of $\tau_{\text {long }}$ for HiPco SWNTs studied in aqueous gels. (Please note the red line is a guide to the eye).

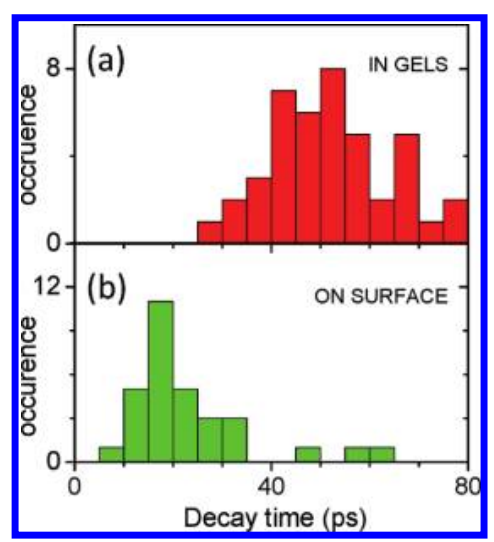

Figure 3. PL decays of HiPco $(6,4)$ SWNTs: (a) Histogram including both the short time $\tau$ for SWNTs studied in aqueous gels displaying biexponential PL decays and the single decay time $\tau$ for those displaying monoexponential decays (90\% of total); (b) histogram of the decay time $\tau$ for SWNTs studied on surfaces (all of them displayed monoexponential decays).

23 ps. This latter distribution is similar to that reported for $(6,4)$ CoMoCat nanotubes on surfaces. ${ }^{5}$

The origin of the biexponential decay was previously attributed to the presence of two closely lying dark $|\mathrm{D}\rangle$ and bright $|B\rangle$ excitonic states (see Figure $4 a$ ) with recombination rates $\Gamma_{\mathrm{D}}$ (purely nonradiative) and $\Gamma_{\mathrm{B}}$ (mainly nonradiative).$^{10}$ Weak PL yields of the bright state (limited to a few percent ${ }^{10,16-19}$ ) are due to nonradiative relaxation pathways (occurring in tens of picoseconds) which are much faster than the radiative recombination time (in the nanoseconds range). Thermalization 


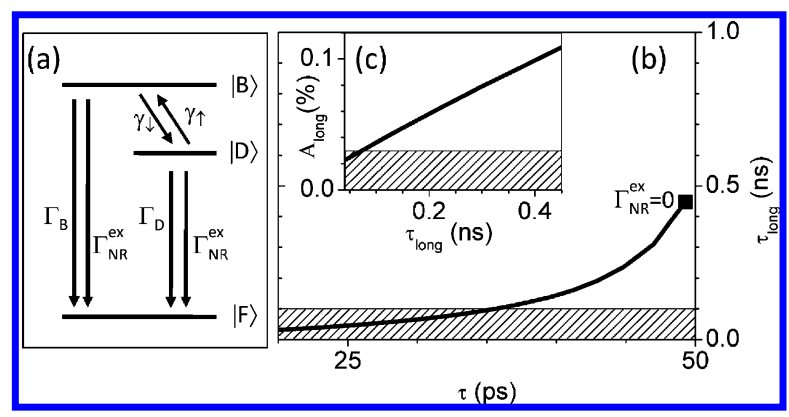

Figure 4. (a) Schematics of the three level model used for simulations (see text). (b) Theoretical long decay times $\tau_{\text {long }}$ as a function of short ones, $\tau$, deduced from simulated PL decays for $\Gamma_{\mathrm{NR}}^{\mathrm{ex}}$ varying from 0 to $30 \mathrm{~ns}^{-1}$. The data point corresponding to $\Gamma_{\mathrm{NR}}^{\mathrm{ex}}=0$ is indicated on the figure. (c) Corresponding long time component fractional yield $A_{\text {long }}$ as a function of $\tau_{\text {long. }}$. The hatched areas represent the domains where $\tau$ and $\tau_{\text {long }}$ are experimentally indistinguishable and $A_{\text {long }}$ extremely small.

between dark and bright states can be promoted through coupling to acoustic phonon modes whose energies match the dark-bright excitonic splitting $\Delta E \approx 5 \mathrm{meV} \cdot{ }^{20,21}$ The transition rates for downhill and uphill processes are $\gamma_{\downarrow}=\gamma_{0}(n+1)$ and $\gamma_{\uparrow}=\gamma_{0} n$, respectively, where $\gamma_{0}$ is the zero temperature bright to dark transition rate ${ }^{22}$ and $n$ is the Bose-Einstein phonon number. ${ }^{10}$

Two mechanisms have been proposed to interpret the exciton fast nonradiative decay process in nanotubes. ${ }^{12}$ The first one involves an enhanced multiphonon decay of localized excitons in potential fluctuations due to the local environment of the nanotubes. The second one is a phonon-assisted indirect exciton ionization process due to the presence of the free carriers which can create a phonon and an intraband electron-hole pair. Importantly, higher defect densities and local perturbations along the nanotubes make both mechanisms more efficient. By solving the kinetic equations, we derived the dynamics of the bright excitonic state population $P_{\mathrm{B}}(t)$ after excitation at $t=0$ (see Supporting Information). The average biexponential behavior of nearly pristine nanotubes (e.g., nanotubes in gels in Figure 2a) can be reproduced assuming $\Gamma_{\mathrm{B}}=20 \mathrm{~ns}^{-1}, \Gamma_{\mathrm{D}}=2 \mathrm{~ns}^{-1}, \gamma_{0}$ $=0.05 \mathrm{~ns}^{-1}$, and an equal population of the bright and dark excitonic states at $t=0$ : one obtains $\tau=50 \mathrm{ps}$ and $\tau_{\mathrm{L}}=450$ ps and a long time component fractional yield $A_{\text {long }}=11 \%$. The small value of $\gamma_{0}$, corresponding to a bright-to-dark transition probability $\sim 400$ times weaker than that of the bright excitonic state recombination, clearly shows that branching to dark states is not the main cause for low PL quantum yield (for the present materials) in agreement with ref 12 .

Typical values for the rates $\gamma_{\uparrow}$ and $\gamma_{\downarrow}$ are orders of magnitude smaller than the nonradiative decay rates $\Gamma_{\mathrm{B}}$ and $\Gamma_{\mathrm{D}}$. Therefore the measured decay times $\tau$ and $\tau_{\text {long }}$ are primarily determined by these rates. The situation is different for the fractional amplitude $A_{\text {long. }}$. Its magnitude is mainly determined by $\gamma_{0}$ and to a lesser extent also by the initial population of the bright and dark states (see Supporting Information).

For nanotubes subject to defects or environmental effects, an additional extrinsic fast nonradiative decay rate, $\Gamma_{\mathrm{NR}}^{\mathrm{ex}}$, should be added to $\Gamma_{\mathrm{B}}$ and $\Gamma_{\mathrm{D}}$. Parts $\mathrm{b}$ and $\mathrm{c}$ of Figure 4 shows the theoretical evolution of $\tau_{\text {long }}$ as a function of $\tau$ and that of $A_{\text {long }}$ as a function of $\tau_{\text {long }}$ when $\Gamma_{\mathrm{NR}}^{\mathrm{ex}}$ varies from 0 to $30 \mathrm{~ns}^{-1}$. The hatched areas represent the domains where $\tau$ and $\tau_{\text {long }}$ are experimentally indistinguishable and where $A_{\text {long }}$ is extremely small. In these cases, the experimental PL decays are well reproduced with a monoexponential curve. For example, for a typical rate of ${ }^{12} \Gamma_{\mathrm{NR}}^{\mathrm{ex}}=20 \mathrm{~ns}^{-1}$ added to the previous values of
$\Gamma_{\mathrm{B}}$ and $\Gamma_{\mathrm{D}}$, the exciton recombination dynamics becomes fast and monoexponential with a decay time $\tau=25 \mathrm{ps}$ in agreement with our experimental observation.

In Figure 4 and from our analysis we identify the involved dark state as the even parity $E_{1}^{1 g}$ located $\sim 5 \mathrm{meV}$ below the bright $s t a t e E_{11}$. In addition, there are two degenerate finite momentum states ( $\mathrm{K}$ and $\mathrm{K}^{\prime}$-momentum excitons) with $\Delta E \approx$ $36 \mathrm{meV}$ for the $(6,5)$ SWNT above the bright one. ${ }^{23,24}$ Direct optical excitation of this state is forbidden, but it can be populated via a phonon sideband located at $\left(\sim E_{11}+200 \mathrm{meV}\right.$ $\left.\approx E_{19}^{19}+170 \mathrm{meV}\right){ }^{23,25}$ Hertel et al. found that after resonant $E_{22}$ excitation a large fraction of the $E_{22}$ excitons decay into this $\mathrm{K}$ momentum state. ${ }^{26}$ According to our model, a large initial population of the dark state is a prerequisite for a detectable fractional amplitude $A_{\text {long }}$ and thus for observing the biexponential decay. To test if these higher-lying dark states influence the PL dynamic significantly, we recorded PL decays at two different excitation wavelengths. One was chosen to be close to the exciton-phonon state at $834 \mathrm{~nm}$ and the other well below at $920 \mathrm{~nm}$. Interestingly, switching between the two excitation wavelengths does not change the decay dynamics substantially: only small changes in the fractional amplitudes and decay times have been observed (data not shown). This would suggest that the K-momentum exciton is not significantly involved in the biexponential decay.

Luminescence decays of $(6,4)$ SWNTs with large decay times $(t>50 \mathrm{ps})$ that do not show biexponential decays cannot be reproduced by only modifying the extrinsic rate $\Gamma_{\mathrm{NR}}^{\mathrm{ex}}$ and keeping the other parameters the same as for $(6,5)$ SWNTs. The experimental observation can be explained within our threelevel model by a reduced $\gamma_{\downarrow}$ between the two states in the case of $(6,4)$ nanotubes. This would be expected due to a larger bright-dark splitting energy $\Delta E$ that was found to scale inversely with the diameter squared. ${ }^{27}$ Keeping all other parameters constant, a small increase in $\Delta E$ based on the different nanotube diameters ( $0.74 \mathrm{vs} 0.68 \mathrm{~nm})$ would lead to a reduction of $A_{\text {long }}$ by a factor of $\sim 2$, and thus the long time component of the decays would be close to the detection limit.

In this work, we have identified the synthesis methods and environmental conditions leading to the observation of monoor biexponential PL decays in individual small diameter SWNTs. A simple model based on a three-level system reproduces the experimental observations taking into account the predominant defect dependent nonradiative decay mechanisms proposed recently. ${ }^{12}$ This work emphasizes the importance of SWNTs processing and observation conditions for obtaining high quality luminescent nanotubes.

Acknowledgment. This research was funded by the Région Aquitaine, the Agence Nationale pour la Recherche (ANR PNANO), the NSF Center for Biological and Environmental Nanotechnology (EEC-0118007 and EEC-0647452), and the Welch Foundation (Grant C-1668). A.H. and T.G. thank the Deutschen Forschungsgemeinschaft (DFG-Grant HA4405/4-1) and the Nanosystems Initiative Munich.

Supporting Information Available: Three-level model describing the biexponential decay of HiPco SWNTs, and figures depicting amplitude factors, initial bright state population $N$ on the measured decay times and fractional yield amplitudes, transition rates vs decay times, and influence of the bright-dark splitting energy on amplitude. This material is available free of charge via the Internet at http://pubs.acs.org. 


\section{References and Notes}

(1) Lefebvre, J.; Maruyama, S.; finnie, P. Photoluminescence: Science and Applications. In Carbon nanotubes; Jorio, A., Dresselhaus, G., Dresselhaus, M. S., Eds.; Springer-Verlag: Berlin-Heidelberg, 2008; Vol. 111, pp 287.

(2) Weisman, R. B.; Bachilo, S. M. Nano Lett. 2003, 3, 1235.

(3) Cognet, L.; Tsyboulski, D. A.; Rocha, J. D.; Doyle, C. D.; Tour, J. M.; Weisman, R. B. Science 2007, 316, 1465.

(4) Lefebvre, J.; Finnie, P. Nano Lett 2008, 8, 1890.

(5) Gokus, T.; Hartschuh, A.; Harutyunyan, H.; Allegrini, M.; Hennrich, F.; Kappes, M.; Green, A. A.; Hersam, M. C.; Araujo, P. T.; Jorio, A. Appl. Phys. Lett. 2008, 92, 153116.

(6) Tsyboulski, D. A.; Bakota, E. L.; Witus, L. S.; Rocha, J.-D. R.; Hartgerink, J. D.; Weisman, R. B. J. Am. Chem. Soc. 2008, 130, 17134. (7) Duque, J. G.; Pasquali, M.; Cognet, L.; Lounis, B. ACS Nano 2009, 3, 2153.

(8) Carlson, L. J.; Krauss, T. D. Acc. Chem. Res. 2008, 41, 235.

(9) Hagen, A.; Steiner, M.; Raschke, M. B.; Lienau, C.; Hertel, T. Qian, H.; Meixner, A. J.; Hartschuh, A. Phvs. Rev. Lett. 2005, 95, 197401 (10) Berciaud, S.; Cognet, L.; Lounis, B. Phvs. Rev. Lett. 2008, 101, 077402

(11) Hogele, A.; Galland, C.; Winger, M.; Imamoglu, A. Phvs. Rev. Lett. 2008, 100, 217401.

(12) Perebeinos, V.; Avouris, P. Phvs. Rev. Lett. 2008, 101, 057401

(13) Xiao, Y. F.; Nhan, T. Q.; Wilson, M. W. B.; Fraser, J. M. Phvs. Rev. Lett. 2010, 104, 017401.
(14) Becker, W. Spring. Ser. Phys.Chem. 2005, 81.

(15) Hartschuh, A.; Pedrosa, H. N.; Novotny, L.; Krauss, T. D. Science 2003, 301, 1354 .

(16) Crochet, J.; Clemens, M.; Hertel, T. J. Am. Chem. Soc. 2007, 129, 8058 .

(17) Carlson, L. J.; Maccagnano, S. E.; Zheng, M.; Silcox, J.; Krauss, T. D. Nano Lett. 2007, 7, 3698.

(18) Tsyboulski, D. A.; Rocha, J. D. R.; Bachilo, S. M.; Cognet, L.; Weisman, R. B. Nano Lett. 2007, 7, 3080.

(19) Lefebvre, J.; Austing, D. G.; Bond, J.; Finnie, P. Nano Lett. 2006, 6, 1603.

(20) Mortimer, I. B.; Nicholas, R. J. Phys. Rev. Lett. 2007, 98.

(21) Shaver, J.; Kono, J.; Portugall, O.; Krstic, V.; Rikken, G.; Miyauchi, Y.; Maruyama, S.; Perebeinos, V. Nano Lett. 2007, 7, 1851.

(22) Perebeinos, V.; Tersoff, J.; Avouris, P. Nano Lett. 2005, 5, 2495.

(23) Torrens, O. N.; Zheng, M.; Kikkawa, J. M. Phys. Rev. Lett. 2008, 101,157401

(24) Murakami, Y.; Lu, B.; Kazaoui, S.; Minami, N.; Okubo, T.; Maruyama, S. Phvs Rev B 2009, 79, 195407.

(25) Berciaud, S.; Cognet, L.; Poulin, P.; Weisman, R. B.; Lounis, B. Nano Lett. 2007, 7, 1203.

(26) Hertel, T.; Perebeinos, V.; Crochet, J.; Arnold, K.; Kappes, M.; Avouris, P. Nano Lett. 2008, 8, 87.

(27) Capaz, R. B.; Spataru, C. D.; Ismail-Beigi, S.; Louie, S. G. Phvs Rev B 2006, 74, 121401.

JP1049217 